\title{
Using Kinect v2 Combined with Unity3D to Design an Agility Training Game
}

\author{
Ya-Shu Kang, Shao-Ting Lu, Chun-Chia Chiu, Chia-Chun Tu, Zhi-Yu Wu, and Yao-Jen Chang
}

\begin{abstract}
In this study, we used Kinect v2 technology along with a skeleton tracking technology to create a training system for students with an agility impairment. Through this system, training can be conducted through exercise games without using a playground. We designed a track and simulated real-world obstacles by using animated games. A single case research method was used to conduct the experiment. Three elementary school children were recruited as participants. Data showed that the time taken to complete the exercises decreased among all the three participants. Social validity results showed the parents and teachers considered the video game useful and it helped their children increase agility effectively.
\end{abstract}

Index Terms-Agility training, exercise games, gamification, Kinect v2.

\section{INTRODUCTION}

Students with an agility impairment usually lack cognitive and learning abilities. They need repetitive training and partial assistance for daily living. This training can improve the students' responsiveness and attentiveness. This study developed a training system based on Kinect v2; this system can overcome not only workplace (setting) problems but also difficulties related to teachers' teaching burden. In particular, Kinect v2 increases the motivation for learning.

To test our system and determine whether it can improve students' agility skill effectiveness, we invited three students with agility impairment to participate in our study.

\section{GAME DESIGN}

In this study, the game was designed in accordance with the degree of physical activity of the students, which can be divided into high and low. This study focused on the use of Microsoft's Kinect v2 skeleton detection and Unity3D platform to establish a training system for students who

Manuscript received February 25, 2019; revised April 29, 2019. This work was supported in part by the National Science Council, Taiwan under Grant 107-2221-E-033 -037.

Ya-Shu Kang is with Department of Special Education, Chung Yuan Christian University, Taoyuan, 320, Taiwan (e-mail: yashu@ cycu.edu.tw).

Chun-Chia Chiu was with Department of Electronic Engineering, Chung Yuan Christian University, Taoyuan, 320, Taiwan (e-mail: spp12026143@cycu.org.tw).

Shao-Ting Lu was with Communication Engineering Master's Programs, Chung Yuan Christian University, Taoyuan, 320, Taiwan (e-mail: milk.t13@gmail.com).

Chia-Chun Tu and Yao-Jen Chang are with Department of Electronic Engineering, Chung Yuan Christian University, Taoyuan, 320, Taiwan (e-mail: chiachun0927@icloud.com,yjchang@cycu.edu.tw).

Zhi-Yu Wu is with Communication Engineering Master's Programs, Chung Yuan Christian University, Taoyuan, 320, Taiwan (e-mail: zhiyu@cycu.org.tw). needed agility improvement. After discussing with the teachers of a primary school, we found that the students had low motivation for repetitive training patterns and low attentiveness, which affected their physical movements.

The game-based intervention phase occurred only in the special education classroom. The Kinect V2 sensor requires an area of 15 feet by 9 feet for motion capture of the player. Within this area furniture should be removed.

The proposed system detects body skeleton information to determine the movement of the whole body. The Kinect v2 depth sensor range is $0.5-4.5 \mathrm{~m}$. Therefore, the system features a systematic correction mechanism to determine whether the participant is in the detection scope of the game; if not, the scope is adjusted.

The incorporation of gesture recognition technology prevents the children from having to wear intrusive body sensors; additionally, Kinect provides real-time three-dimensional (3D) anatomical landmark position data, and it is inexpensive, portable, and simple to set up. Studies have also shown that the Microsoft Kinect and 3D motion analysis systems have comparable intertrial reliability and excellent concurrent validity [1]-[3]. The motion capture capability of Kinect makes it a candidate for implementing assisitive technology targeting students with intellectual disabilities (ID) and other disabilities to improve their social behaviors [4], engage students in vocational training [5], [6], provide leisure time opportunities [7], detect stereotypical motor movements [8], [9], and facilitate physical rehabilitation [3], [10]-[13].

Our study uses Kinect V2 in the development of interactive games for children with ID to encourage engagement in agility training. The purpose of this study was to examine whether game technology is an effective way to teach elementary students with ID to improve agility, and to determine the long-term maintenance effects of using game technology in the agility training.

To ensure that the participant is within the detection range during the training session, accurate detection of the joint coordinates is required. To begin the game, the students must raise their hands to start playing; raising the hand indicates that the students is ready and can thus begin the game. The game screen was built using the Unity3D platform, and the game is modeled as a 3D game, rather than a $2 \mathrm{D}$ game as in previous systems, to ensure that the students can have a more realistic perception (Fig. 1). Through this method, the students could link the characters on the screen (Fig. 2).

\section{EXPERIMENT DESIGN}

This study tested the proposed system in an urban special 
education class, which receives students with special needs from kindergarten through elementary school. The Kinect V2 device was connected to a Lenovo Thinkpad T61 notebook computer, and the game software developed in-house was installed with Microsoft Windows 7. The computer had an audio module, which we used to deliver audio feedback, and an external 22-inch LCD screen that was used for visual interaction. The software was coded using the Unity 3D software and the Microsoft Visual Studio software development environment tools to form an integrated development platform. The interactive interface with audio and video feedback was programmed to reinforce children's motivation to engage in the training. For optimal performance of the Kinect V2 sensor, the participants were required to stand approximately 3 feet in front of the Kinect V2 module (Fig. 2).

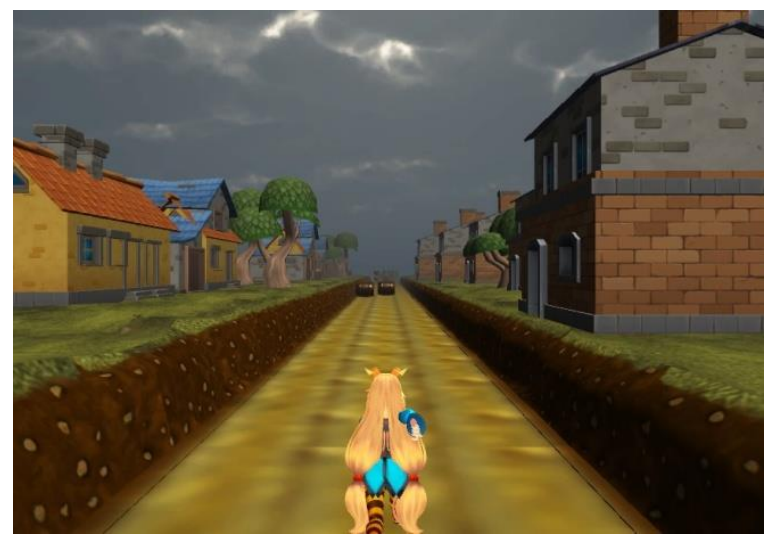

Fig. 1. Agility training game.

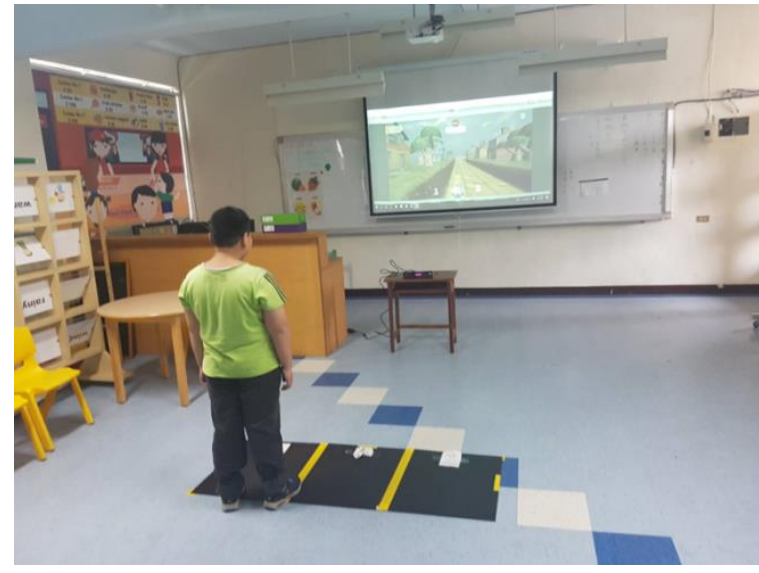

Fig. 2. Video game for participants with special needs.

We held a discussion with the children's parents regarding the benefits of the training system for the students, after which the parents agreed to sign the partial test consent form. The participants of the experiment were students with three levels of impairments, as given in Table I. To protect the privacy of the participants, only the first names of the students are provided.

Each game was played three times for a duration of $3 \mathrm{~min}$, and the students' results appeared at the end of the game. Examples of the results include number of lanes, squatting times, total number of miles, and number of cakes. To prevent the students from deliberately hitting the wooden box, the system was set to end of the game when the player hit the box three times. We used a single case design methodology, which was divided into a baseline (baseline), an intervention period (intervention), and a maintenance phase (maintenance) [11].

\begin{tabular}{llll}
\multicolumn{3}{c}{ TABLE I: PROFILES OF PARTICIPANTS } \\
\hline \hline Names & Gender & Age (Years) & Disabilities \\
\hline Alex & Boy & 9 & $\begin{array}{l}\text { Intellectual } \\
\text { Disability } \\
\text { (moderate) } \\
\text { Brian }\end{array}$ \\
& Boy & 10 & $\begin{array}{l}\text { Intellectual } \\
\text { Disability } \\
\text { (Severe) } \\
\text { Intellectual }\end{array}$ \\
Cindy & Girl & 8 & $\begin{array}{l}\text { Disability } \\
\text { (moderate) }\end{array}$ \\
\hline \hline
\end{tabular}

To assess the ability of the agile training game in helping students who needed agility improvement, this experiment focused on the steps that are typically taken by primary school teachers and physiotherapists. Thus, a distance of $20 \mathrm{~m}$ was set for picking up objects (picking up objects eight times in the squatting position) and zigzag runs; hence, this experiment focused on the picking up of things (Fig. 3).

According to Alex's teacher, although his physical ability is not bad, his agile response and attention requires improvement. Brian had a difficulty understanding oral instructions. He also had problems generalizing the lane in the game to the actual lane on the playground. Therefore, we decided to label the lanes in the game with numbers $1,2,3$ to help him understand the lanes in the game were just like the numbered lanes on the playground. Cindy got distracted easily. Therefore, she usually needed extraordinarily long time to finish a task. Computer games was expected to help her keep concentrated in our experiment.

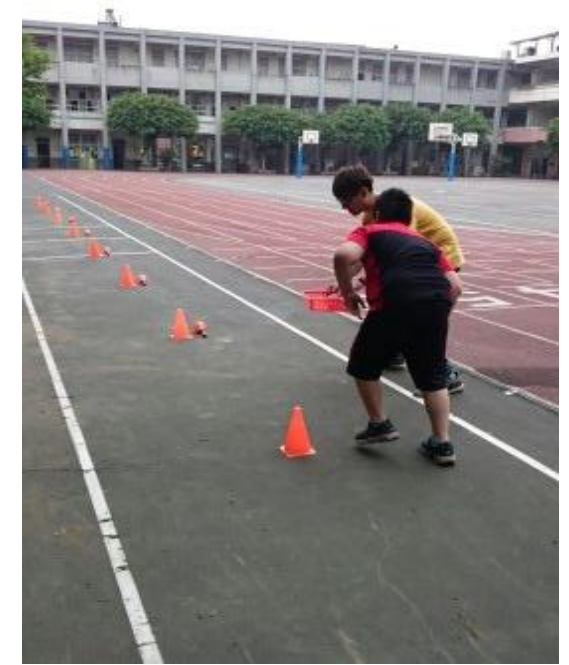

Fig. 3. Assessment of movement agility.

\section{EXPERIMENT RESULTS}

This study assessed the effectiveness of the proposed system with regard to elementary-age children receiving agility training. The completion time for each task is presented in Fig. 4, Fig. 5, and Fig. 6. Overall, the children's independent performance in terms of task completion time immediately decreased when the game intervention was introduced, and all of the participants acquired and 
maintained the skills necessary for the task executed independently. During the baseline phase, Alex completed a mean of $37 \mathrm{~s}$ for independent agility training; during the maintenance phase, his performance improved to $20 \mathrm{~s}$. In other words, Alex completed the picking task in an average of approximately $20 \mathrm{~s}$, substantially improving his picking efficiency. In the gameplay, Alex was also trained to dodge a wooden box. However, he sometimes deliberately hit the wooden box that caused the game to end very quickly. Brian's mean task completion time during the baseline phase was $52 \mathrm{~s}$; Brian maintained $30 \mathrm{~s}$ for completing the task during the maintenance phase. Cindy mean task completion time during the baseline phase was $64 \mathrm{~s}$. During the maintenance phases, her mean task completion time decreased to $27 \mathrm{~s}$.

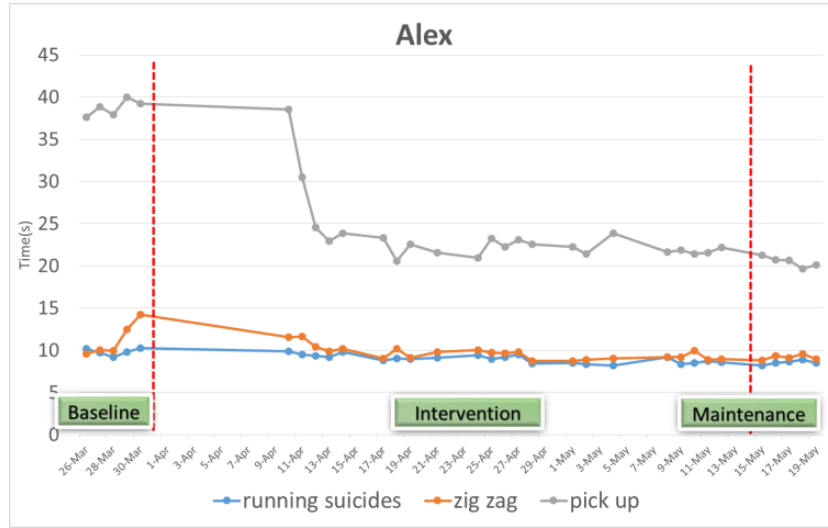

Fig. 4. Task completion times for Alex.

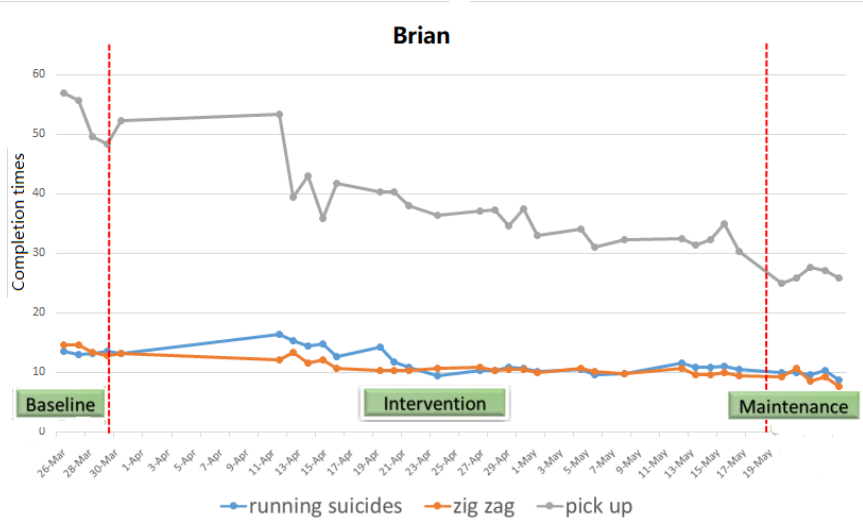

Fig. 5. Task completion times for Brian.

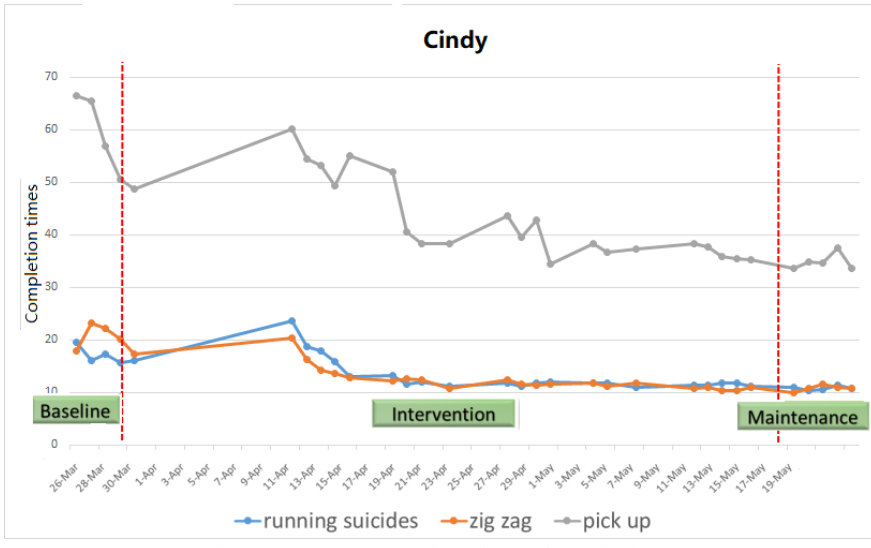

Fig. 6. Task completion times for Cindy.

Social validity was evaluated through a survey, which assessed the participants' experience and success during the study. The survey was administered during a brief interview to each child's parents, with questions analyzing personal opinions regarding practical use of the video game. Open-ended and closed questions examined the parents' general thoughts regarding the video game and asked whether the game had helped their children acquire agility. Other questions assessed whether the game changed the method by which each participant performed the task independently. Parents were also asked whether they would like to have their children participate in the future and what tasks would be helpful for their children to learn. Finally the interview addressed whether they had discussed the video game with anyone outside of school. One researcher asked each participant's parents the questions, and another audio recorded the verbal responses. Another survey was distributed to each teacher as a hard copy on which to write responses. These questions evaluated the participants' satisfaction and their perception of whether the video game was acceptable and useful with students.

The parents thought the video game was very useful and it had helped their children acquire agility effectively. The game also changed the method by which their children performed the task independently. Additionally, parents would like to have their children participate in the future and have shopping skills and pedestrian safety listed in the game-based interventions also. Finally, parents discussed outside of school the video game with their friends who also had children with ID. They highly recommended the game and encouraged their friends to contact the teachers for more information.

According to the teachers, participants enjoyed playing the video game and found the game to be an enjoyable motivator. The teachers agreed that the video game improved independence and make teaching easier. The teachers expressed a wish for more time and resources to create additional video games for their students. They also found the video game to be up-to-date with current teaching methods. Teachers would like to use this method with their other students to reduce direct verbal prompting and other individual instruction. The teachers commented that education should include more technology where possible.

\section{DISCUSSIONS}

In this training system based on Kinect v2, the user's skeleton information must be determined first by using the skeleton tracking function so that the user's whole body can be used to execute the game. Nevertheless, the presence of any foreign objects in front of the lens can result in difficulties in the skeleton tracking. For example, if other individuals enter the Kinect v2 detection range, the user skeleton information would be lost. Because there is no solution at present to overcome this problem on the Unity3D platform, the test must be conducted in open and small places. In addition, because the system is currently only for squatting and left and right mobile training, for Kinect v2 to determine the user's jump, the user must lift his or her feet or jump a certain height. However, because the jumping action is considerably difficult for children to perform, after discussion 
with the primary school teacher, jump training was not included in this study.

As this and other studies have demonstrated, even simple tasks can present complex challenges for some individuals with ID. Organizing tasks into a sequence of smaller steps or actions is an evidence-based practice for teaching new skills to students with severe developmental disabilities [14]. Moreover, having an understanding of all the steps involved for a particular task can assist in identifying those steps that require extra training and can help teach tasks in a logical progression [15]. The results of the present study show that the proposed game helped children with ID familiarize themselves with all the task steps involved in washing their hands independently and, in particular, assisted them with the task steps where they needed extra practice.

\section{CONCLUSIONS}

After discussing with the teacher, we decided to use a checklist to assess whether the physical ability of the students improved after using the training system.

We found that after training, the physical ability and efficiency of the students indeed improved. Therefore, this system not only enhances the learning motivation of the students but also detects whether the students' actions are correct. Moreover, the system uses interesting pictures and sounds as feedback to enhance the self-confidence of the students. Thus, using this system for training can effectively reduce the burden on teachers. Because this system is low cost and light in weight, it can also be used at home, and thus, the students can train not only at the school but also at home.

According to the results of this study, science and technology is not only a future trend but can actually help people to have a better quality of life.

\section{REFERENCES}

[1] R. A. Clark, Y. H. Pua, K. Fortin, C. Ritchie, K. E. Webster, L. Denehy, and A. L. Bryant, "Validity of the Microsoft Kinect for assessment of postural control," Gait \& Posture, vol. 36, no. 3, pp. 372-377, July 2012.

[2] T. Dutta, "Evaluation of the Kinect" sensor for 3-D kinematic measurement in the workplace," Applied Ergonomics, vol. 43, no. 4, pp. 645-649, July 2012.

[3] B. Galna, G. Barry, D. Jackson, D. Mhiripiri, P. Olivier, and L. Rochester, "Accuracy of the Microsoft Kinect sensor for measuring movement in people with Parkinson's disease," Gait \& Posture, vol. 39 , no. 4, pp. 1062-1068, Apr. 2014.

[4] N. Uzuegbunam, W. H. Wong, S. C. S. Cheung, and L. Ruble, "MEBook: Kinect-based self-modeling intervention for children with autism," in Proc. 2015 IEEE International Conference on Multimedia and Expo (ICME), 2015, pp. 1-6.

[5] Y. J. Chang, S. F. Chen, and A. F. Chuang, "A gesture recognition system to transition autonomously through vocational tasks for individuals with cognitive impairments," Research in Developmental Disabilities, vol. 32, no. 6, pp. 2064-2068, Nov.-Dec. 2011.

[6] Y. J. Chang, L. D. Chou, F. T. Y. Wang, and S. F. Chen, "A Kinect-based vocational task prompting system for individuals with cognitive impairments," Personal and Ubiquitous Computing, vol. 17, no. 2, pp. 351-358, Feb. 2013.

[7] G. Altanis, M. Boloudakis, S. Retalis, and N. Nikou, "Children with motor impairments play a Kinect learning game: First findings from a pilot case in an authentic classroom environment," Interaction Design and Architecture Journal, no. 19, pp. 91-104, Winter 2013-2014.

[8] N. Gonçalves, J. L. Rodrigues, S. Costa, and F. Soares, "Preliminary study on determining stereotypical motor movements," in Proc. 2012
Annual International Conference of the IEEE Engineering in Medicine and Biology Society (EMBC), 2012, pp. 1598-1601.

[9] T. W. Wei, "Detecting the hand-mouthing behavior of children with intellectual disability using Kinect imaging technology," in Proc. of the 14th International ACM SIGACCESS Conference on Computers and Accessibility, 2012, pp. 295-296.

[10] Y. J. Chang, S. F. Chen, and J. D. Huang, "A Kinect-based system for physical rehabilitation: A pilot study for young adults with motor disabilities," Research in Developmental Disabilities, vol. 32, no. 6, pp. 2566-2570, Nov.-Dec. 2011.

[11] Y. J. Chang, W. Y. Han, and Y. C. Tsai, "A Kinect-based upper limb rehabilitation system to assist people with cerebral palsy," Research in Developmental Disabilities, vol. 34, no. 11, pp. 3654-3659, Nov. 2013.

[12] J. W. Hung, Y. J. Chang, and W. Y. Han, "Game technology to increase range of motion for adolescents with cerebral palsy: A feasibility study," International Journal on Disability and Human Development, vol. 16, no. 3, pp. 267-274, Aug. 2017.

[13] L. Luna-Oliva, R. M. Ortiz-Gutiérrez, R. C. Cuerda, R. M. Piédrola, I. M. Alguacil-Diego, C. Sánchez-Camarero, and M. D. C. Martínez Culebras, "Kinect Xbox 360 as a therapeutic modality for children with cerebral palsy in a school environment: A preliminary study," Neuro Rehabilitation, vol. 33, no. 4, pp. 513-521, 2013.

[14] F. Spooner, V. Knight, D. Browder, and B. Smith, "Evidence based practices for teaching academic skills to students with severe developmental disabilities," Remedial and Special Education, vol. 33, no. 6, pp. 374-387, 2012.

[15] J. O. Cooper, T. E. Heron, and W. L. Heward, Applied Behavior Analysis, 2nd ed. Upper Saddle River, NJ: Pearson Merrill Prentice Hall, 2007.

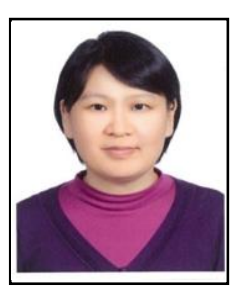

Ya-shu Kang is an assistant professor in Department of Special Education at Chung Yuan Christian University. Kang received her Ph.D. from the University of Oklahoma, and has been involved in special education for over 10 years. At CYCU, she teaches and conducts research in the area of learning disabilities, inclusive education, early intervention, education instructional design, and educational technology for students with special needs. She also serves as the member of special education committee. She can be contacted atyashu@cycu.edu.tw.

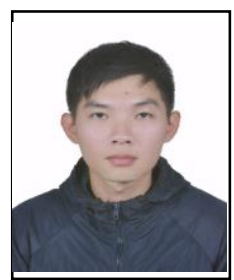

Chun-Chia Chiu received his master degree from Department of Electronic Engineering, Chung Yuan Christian University in 2018. His major research areas include games for children with autism spectrum disorder to help children engage in repetitive practice through the exergaming using Kinect and Unity. He can be contacted at spp12026143@ @ycu.org.tw.

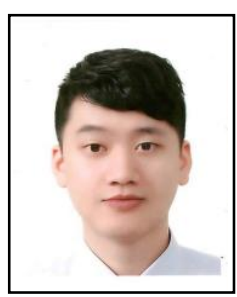

Shao-Ting Lu graduated from Communication Engineering Master's Programs, Chung Yuan Christian University in 2017. His major research areas include games for children with intellectual and developmental disabilities to help children engage in repetitive practice through the exergaming using Kinect and Unity. He can be contacted at milk.t13@gmail.com.

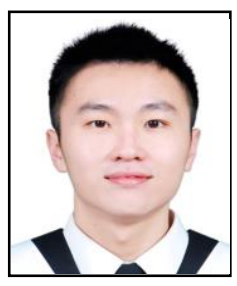

Chia-Chun Tu graduated from Department of Electronic Engineering, Chung Yuan Christian University in 2017. He is studying in the second year of Electronic Engineering Master's degree program in Chung Yuan Christian University. His major research areas include health promotion games for children with intellectual and developmental disabilities to help children engage in repetitive practice through the exergaming using Kinect and Scratch. He can be contacted at chiachun0927@icloud.com. 


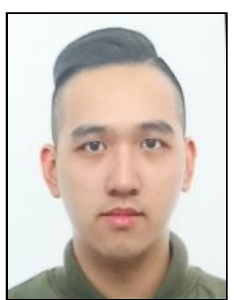

Zhi-Yu Wu graduated from Department of Electronic Engineering, Chung Yuan Christian University in 2018. He is studying in the first year of the Communication Engineering Master's Programs in Chung Yuan Christian University. His research interest is on gaming technology for children with special needs. He can be contacted at zhiyu@ cycu.org.tw.

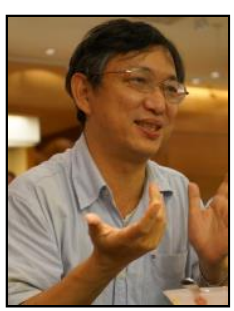

Yao-Jen Chang received his bachelor degree and PhD degree from Department of Electrical Engineering, National Taiwan University, in 1986 and 1992, respectively. He was a visiting research professor at University of Illinois at Urbana-Champaign from 1992 to 1995. Dr. Chang joined Department of Electronic Engineering, Chung Yuan Christian University, Taiwan, in 1995. He has been working on mobile computing and electronic commerce since then. He took a leave to Yam Digital as a chief scientist from 2000 to 2006. Professor Chang has refocused his research in disability study and assistive technology since 2007 . He started to serve as the chairman of Association of Supported Employment for People with Disabilities in 2009. Dr. Chang has strong research partnership with major medical institutions and non-profit organizations in Taiwan. For his pioneering work and commitment to research in disabilities, he was promoted as a distinguished professor in 2015. He can be contacted at yjchang@cycu.edu.tw. 